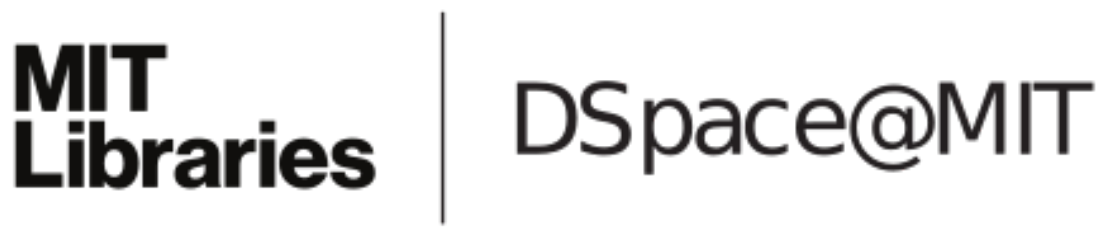

\author{
MIT Open Access Articles
}

Predictability in a hydrodynamic pilot-
wave system: Resolution of walker tunneling

The MIT Faculty has made this article openly available. Please share how this access benefits you. Your story matters.

As Published: 10.1103/PHYSREVE.102.013104

Publisher: American Physical Society (APS)

Persistent URL: https://hdl.handle.net/1721.1/135251

Version: Final published version: final published article, as it appeared in a journal, conference proceedings, or other formally published context

Terms of Use: Article is made available in accordance with the publisher's policy and may be subject to US copyright law. Please refer to the publisher's site for terms of use. 


\title{
Predictability in a hydrodynamic pilot-wave system: Resolution of walker tunneling
}

\author{
Loïc Tadrist $\odot^{*}$ and Tristan Gilet \\ Microfluidics Lab, Aerospace and Mechanical Engineering, University of Liege, Allée de la découverte 9, 4000 Liège, Belgium \\ Peter Schlagheck \\ IPNAS, CESAM research unit, University of Liege, Allée du 6 Août 15, 4000 Liège, Belgium \\ John W. M. Bush \\ Department of Mathematics, Massachusetts Institute of Technology, Cambridge, Massachusetts 02139, USA
}

(Received 20 May 2019; revised 30 March 2020; accepted 21 June 2020; published 9 July 2020)

\begin{abstract}
A walker is a macroscopic coupling of a droplet and a capillary wave field that exhibits several quantumlike properties. In 2009, Eddi et al. [Phys. Rev. Lett. 102, 240401 (2009)] showed that walkers may cross a submerged barrier in an unpredictable manner and named this behavior "unpredictable walker tunneling." In quantum mechanics, tunneling is one of the simplest arrangements where similar unpredictability occurs. In this paper, we investigate how unpredictability can be unveiled for walkers through an experimental study of walker tunneling with precision. We refine both time and position measurements to take into account the fast bouncing dynamics of the system. Tunneling is shown to be unpredictable until a distance of $2.6 \mathrm{~mm}$ from the barrier center, where we observe the separation of reflected and transmitted trajectories in the position-velocity phase-space. The unpredictability is unlikely to be attributable to either uncertainty in the initial conditions or to the noise in the experiment. It is more likely due to changes in the drop's vertical dynamics arising when it interacts with the barrier. We compare this macroscopic system to a tunneling quantum particle that is subjected to repeated measurements of its position and momentum. We show that, despite the different theoretical treatments of these two disparate systems, similar patterns emerge in the position-velocity phase space.
\end{abstract}

DOI: 10.1103/PhysRevE.102.013104

\section{INTRODUCTION}

A silicone oil droplet can bounce indefinitely on the surface of a vertically vibrated oil bath provided that the vibrational acceleration $\Gamma \cos (\Omega t)$ is sufficiently large. When the droplet impacts the oil bath at half the driving frequency $\Omega / 4 \pi$, the resulting surface waves may, under some restrictive conditions, propel the droplet along the bath surface. The dual object composed of the droplet and its accompanying wave field is called a walker [1,2]. Several works have provided a characterization of single walkers from a hydrodynamic point of view [3-7]. The waves surrounding the walker comprise long-lived standing Faraday waves and relatively short-lived traveling capillary waves. The drop acquires horizontal momentum by bouncing on its sloping waves. Walkers exhibit several properties reminiscent of quantum particles, and their dynamics is similar in form to that imagined by Louis de Broglie in his double-solution pilot-wave theory [8].

The trajectory of a walker confined in a potential well or by a Coriolis force self-organizes along quantized periodic orbits [9-12]. In a cavity, a walker moves chaotically, and its emergent statistics is comparable to that calculated from Schrödinger's equation $[13,14]$, provided the Faraday wavelength $\lambda_{F}$ of the walker is identified with the de Broglie wavelength $\lambda_{\mathrm{dB}}$ of the quantum particle [8]. However, the

\footnotetext{
*loic.tadrist@uliege.be; http://lab.tadrist.fr
}

analogy is currently less clear in the case of the doubleslit interference experiment, for which the walker's emergent statistical behavior is neither quantum mechanical nor purely classical [15-18].

In this article, we address the question of unpredictability in an experimental configuration proposed as a macroscopic analog of quantum tunneling [19-21]: a walking droplet is launched toward a submerged boundary that it may cross or not (see Fig. 1). The first experimental demonstration of walker tunneling was done by Eddi et al. [19]. Single Walkers were repeatedly confined in a rhomboidal cavity divided in the middle by a submerged barrier. They showed that the crossing probability (i.e., the number of crossing events divided by the number of crossing attempts) is not a step function of the walker velocity as it would be in classical point mechanics. Nevertheless, the walker dynamics is fully deterministic: the probabilistic description only reflects a limited knowledge of the system [22-25].

This experiment has been analysed theoretically by Hubert et al. [21] and Nachbin [20]. Hubert et al. [21] showed that one possible reason for the "unpredictable tunneling" of walkers was the lack of control of initial parameters such as the droplet velocity (which was controlled at $\pm 5 \%$ in the original experiment) and the incident angle. Nachbin [20] examined experimentally a simplified one-dimensional confined version of walker tunneling and showed that the wave construction in the second cavity may allow the walker to cross the 

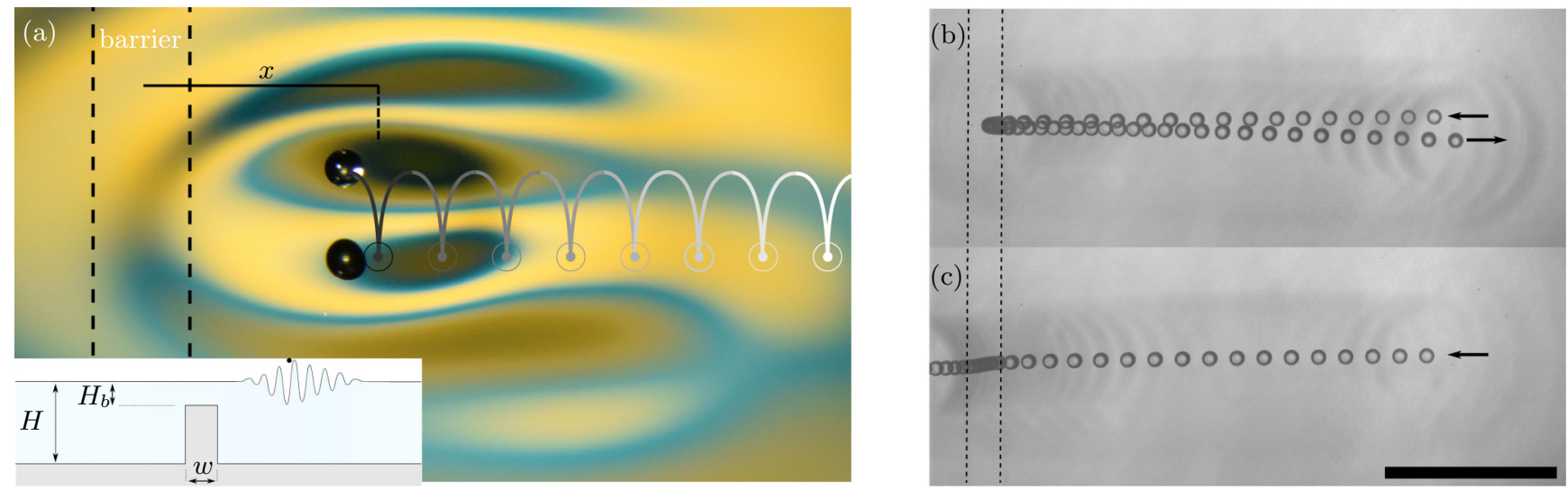

FIG. 1. Illustration of the experiment. (a) Artistic view of a walker sent toward the barrier (not to scale). The barrier is a submerged boundary of width $w$ and depth $H_{b}$ (inset). (b) Snapshots of a reflected trajectory. (c) Snapshots of a transmitted trajectory. Top views of the droplet in panels (b) and (c) are taken at $8 \mathrm{~Hz}$. The scale bar is $1 \mathrm{~cm}$ and the barrier position is shown with dotted lines.

barrier. Both theoretical studies describe walker tunneling, but rationalize the observed unpredictability on different physical grounds, variability of initial parameters or the peculiar buildup of the wave-field before crossing the barrier.

In this paper, we compare the level of noise to the intrinsic sensitivity of the walker tunneling experiment. Is noise responsible for "unpredictable" tunneling? Does unpredictability emerge from the non-Markovian and possibly chaotic dynamics of the walker? To what extent can one predict that the walker will be transmitted or reflected based on some cross section of the phase space? We begin by reproducing walker tunneling in well controlled experimental conditions. Subsequently, we analyze the effects of external parameters and the measurement process on the accuracy of our results. We then describe the intrinsic variability of the walkers and stress the undercharacterization of the system by position and velocity only. Finally we analyze quantum tunneling with the repeated measurement formalism that allows for comparison with the walking droplet system. We show that in both cases, the predictability of the system increases only at the foot of the barrier.

\section{EXPERIMENTAL SETUP}

We refined the walker tunneling experiment initially performed by Eddi et al. [19]. Laser-cut PMMA pieces were glued to the bottom of a reservoir and submerged with oil. Neither swelling nor deformation of the plastic pieces were observed during and after the experiment. The barrier to be tunneled consisted of a thin plastic piece placed at the center of the oil bath. The dimensions of the submerged plastic pieces are reported in Fig. 2. The geometry of the submerged boundaries is different from that reported in Eddi et al. [19]. It ensures that the droplet is far from other boundaries when interacting with the barrier. The liquid depth varied depending on the region considered. Above the circular boundaries (gray region in Fig. 2) and the launching pieces (white regions in Fig. 2), the liquid depth was a few hundred microns, ensuring rapid local wave damping. The depth was $H_{b} \simeq 1.2 \mathrm{~mm}$ above the barrier and $H=6.5 \mathrm{~mm}$ elsewhere, see Table I.
Both bath and drop were composed of silicone oil of viscosity $v=20 \mathrm{cSt}$ at $25^{\circ} \mathrm{C}$, surface tension $\gamma=20 \mathrm{mN} / \mathrm{m}$ and density $\rho=956 \mathrm{~kg} / \mathrm{m}^{3}$ (Table I). The bath was shaken at $\Omega / 2 \pi=80 \mathrm{~Hz}$ which corresponds to a Faraday wavelength $\lambda_{F}=4.75 \mathrm{~mm}$ [7]. Vertical vibrations were produced with a DataPhysics V55 electrodynamic shaker. A rod and an air bearing carriage were fixed on top of the shaker to ensure reduced lateral vibrations (less than $2.5 \%$ of the vertical vibrations) [26]. Vertical accelerations were measured by a pair of miniature accelerometers (PCB 352C65) and feedback controlled with a home-made Labview software. A DAQ (NIUSB-6343) and an amplifier (PA300E) were used to acquire the acceleration signals and drive the shaker, respectively. The generated signal had a variation $\Delta \Omega / \Omega<$ $0.02 \%$ in frequency and the accuracy on shaking acceleration was $\Delta \Gamma=0.002 \mathrm{~g}$, which represents a relative error of $\Delta \Gamma / \Gamma \simeq 0.05 \%$.

The droplet was formed by a piezoactuated droplet dispenser detailed in Ref. [27]. At this step, the droplet simply bounced on top of the bath but did not walk. To eliminate the influence of ambient air currents [17], the top of the bath was sealed with an anti-reflective acrylic lid. This precaution also guarded the droplet from dust, thus minimizing the chance of rogue coalescence events. To further extend the life of the droplet, special care was taken to clean all surfaces in contact with oil when the experiment was set up. In our experiment, the droplet could thus bounce for more than $12 \mathrm{~h}$ without coalescing with the underlying bath.

Prior to experiments, the bath was shaken at $\Omega / 2 \pi=$ $80 \mathrm{~Hz}$ with a shaking acceleration of $\Gamma=4 \mathrm{~g}$ for $2 \mathrm{~h}$, to be warmed up. This prevented any significant thermal drift during the course of the experiments. The Faraday threshold was then measured by increasing $\Gamma$ until Faraday waves appeared at the liquid surface, at $\Gamma_{F} \simeq 4.4 \mathrm{~g}$. The shaking acceleration was then set back to $\Gamma=2 g$.

Subsequently, the shaking acceleration was increased to $\Gamma>3.8 \mathrm{~g}$, beyond the walking threshold. At this point, the droplet walked on the bath surface and followed the path prescribed by the submerged pieces (see Fig. 2). The latter were arranged so as to send the walker normal to a submerged 
(a)

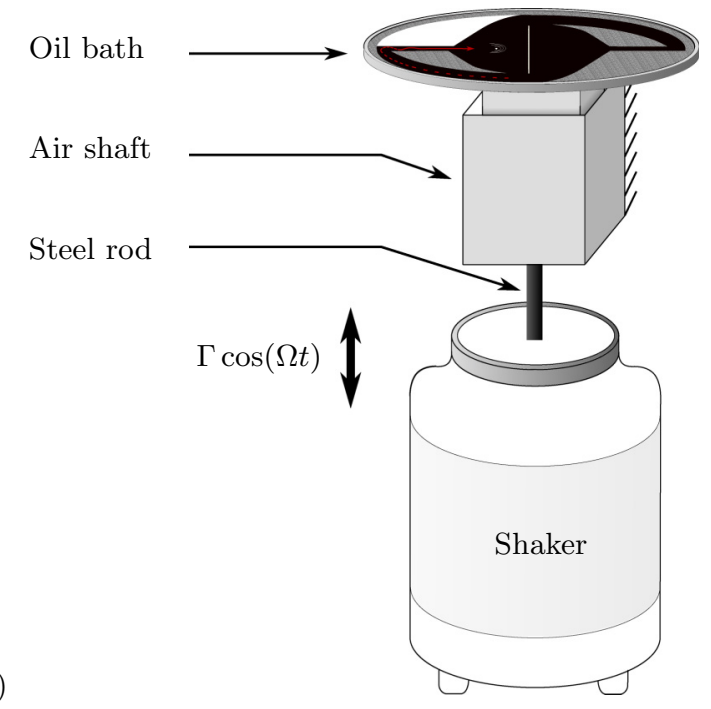

(b)

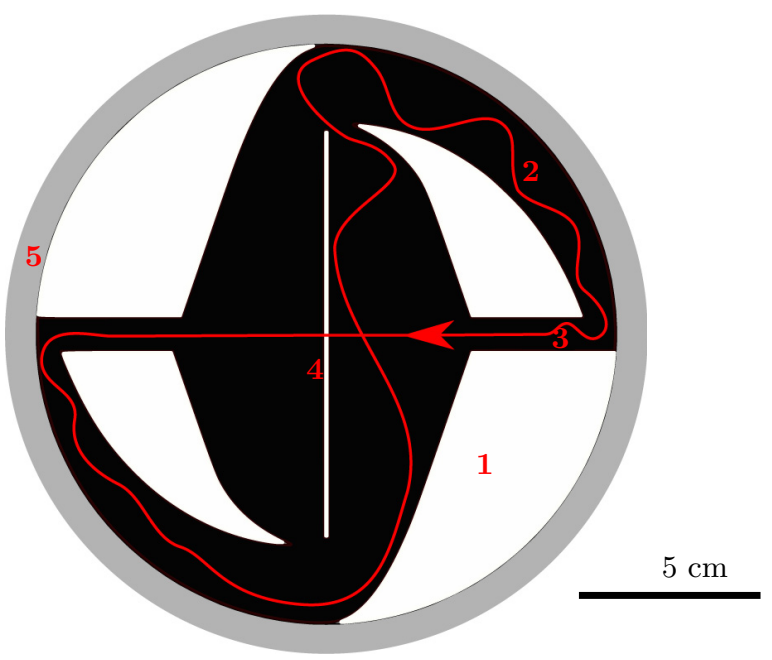

FIG. 2. (a) Experimental setup. (b) Arrangement of the submerged pieces in the bath (Top view) and sketch of a transmitted trajectory. (1) Submerged plastic piece. (2) A narrowing channel guides the droplet toward the launching channel. (3) Launching channel. The droplet oscillates horizontally at the entry then walks straight. (4) Barrier of width $2 \mathrm{~mm}$. (5) Shallow boundary on which waves are strongly damped. The liquid depth was $H=6.5 \mathrm{~mm}$ in the black region, $H_{b}=1.2 \mathrm{~mm}$ on top of the barrier and less than $0.5 \mathrm{~mm}$ above other white and gray regions.

barrier of width $w=2.00 \pm 0.02 \mathrm{~mm}$. The associated waves were highly damped above the barrier since the oil depth $H_{b}$ on the barrier was only a few times the thickness of the viscous boundary layer ( $200 \mu \mathrm{m}$ [7]). The wave-induced speed of the walker was thus reduced by the barrier, which had an effect similar to that of a potential barrier. The impinging walker could either cross the barrier or be reflected. This cavity allowed us to repeatedly send the same walker toward the barrier with a very similar trajectory. After a crossing attempt occurred, the walker moved to the side channels, then to the launching channel where it was again directed toward the barrier for the next crossing attempt.

TABLE I. Table of parameters.

\begin{tabular}{|c|c|c|c|}
\hline & Value & & Parameter \\
\hline$\Omega / 2 \pi$ & $80 \mathrm{~Hz}$ & $\pm 0.02 \%$ & Shaking frequency \\
\hline$\Gamma$ & $4 g$ & $\pm 0.05 \%$ & Shaking acceleration amplitude \\
\hline$\Gamma_{F}$ & $4.4 g$ & $\pm 0.2 \%$ & Faraday threshold \\
\hline$H$ & $6.5 \mathrm{~mm}$ & $\pm 1.5 \%$ & Liquid depth \\
\hline$H_{b}$ & $1.24 \mathrm{~mm}$ & $\pm 2 \%$ & Liquid depth above the barrier \\
\hline$w$ & $2 \mathrm{~mm}$ & $\pm 1 \%$ & Barrier width \\
\hline$R$ & $420 \mu \mathrm{m}$ & $\pm 5 \%$ & Drop radius \\
\hline$V_{w}$ & $12.1 \mathrm{~mm} / \mathrm{s}$ & $\pm 1 \%$ & Free walking speed \\
\hline$\theta_{i}$ & $0.85^{\circ}$ & $\pm 1.2 \%$ & Incident angle \\
\hline$\lambda_{F}$ & $4.75 \mathrm{~mm}$ & & Faraday wavelength \\
\hline$\xi$ & $8.1 \mathrm{~mm}$ & & Wave field damping length \\
\hline$\sigma$ & $20 \mathrm{mN} / \mathrm{m}$ & & Oil surface tension \\
\hline$v$ & $20 \mathrm{cSt}$ & & Oil viscosity \\
\hline$\rho$ & $956 \mathrm{~kg} / \mathrm{m}^{3}$ & & Oil density \\
\hline$v$ & \multicolumn{3}{|c|}{ Instantaneous walking speed } \\
\hline$v_{y}$ & \multicolumn{3}{|c|}{ Instantaneous $y$-axis walking speed } \\
\hline$\theta$ & \multicolumn{3}{|c|}{ Instantaneous trajectory angle } \\
\hline$\phi$ & \multicolumn{3}{|c|}{ Impact phase } \\
\hline$\langle\cdot\rangle$ & \multicolumn{3}{|c|}{ group average } \\
\hline$D_{M}$ & \multicolumn{3}{|c|}{ Demix distance } \\
\hline$\lambda_{\mathrm{dB}}$ & \multicolumn{3}{|c|}{ de Broglie wave length } \\
\hline$\Psi$ & \multicolumn{3}{|c|}{ Shrödinger probability wave } \\
\hline$\chi_{n, l}$ & \multicolumn{3}{|c|}{ Projector function } \\
\hline
\end{tabular}




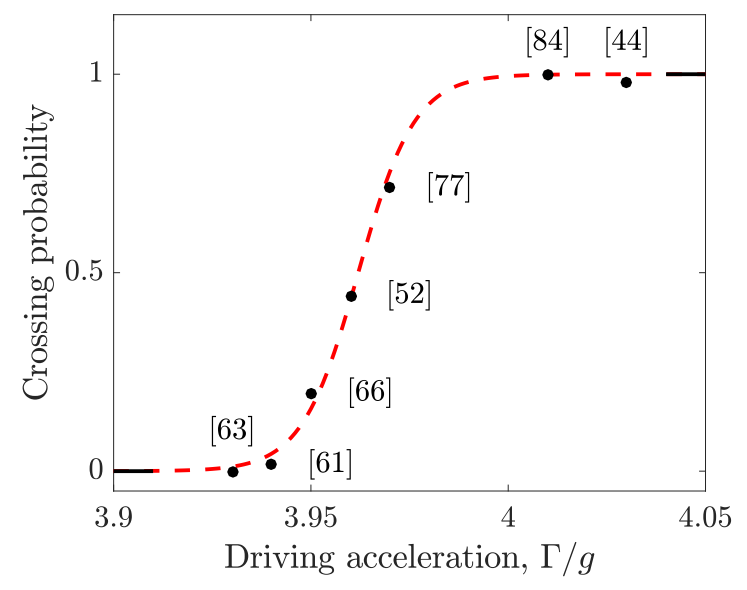

FIG. 3. Transmission probability as a function of shaking acceleration $\Gamma$. The number of launches recorded to establish the probability is reported for each point. The dotted line is a guide for the eyes.

We chose to work with a relatively fast droplet to minimize its transit time around the pool. To avoid any unwanted chaotic bouncing, we considered a droplet walking in the $(2,1)^{2}$ mode for an acceleration $\Gamma \simeq 3.9 \mathrm{~g}$. This fixed the vibration number $\Omega / \sqrt{\gamma / \rho R^{3}}$ at about 0.95 , where $R$ is the drop radius (see Ref. [[8], Fig. 2]). The desired drop diameter was then $2 R \simeq 840 \mu \mathrm{m}$, which set the free walking speed $V_{w}$ in the range $12-14 \mathrm{~mm} / \mathrm{s}$ (see Ref. [[28], Fig. 4.13]).

The proximity to threshold was $\mathcal{M}=\Gamma_{F} /\left(\Gamma_{F}-\Gamma\right) \simeq 7.2$. The associated memory $M_{\mathrm{e}}$ (i.e., the number of bounces that actually compound the wave field) was 5.8, computed with the formula found in Ref. [[7], Eq. (2.65)]. We chose to work in this regime of relatively low memory to limit the spatial extent of the Faraday waves and to ensure that the waves created by the walker in the experimental zone were completely damped during its passage in the side channel. The damping length of the waves computed with Ref. [[7], Eq. (2.60)] is $\xi=8.1 \mathrm{~mm}$ in front of the droplet $(\theta=0), \xi=8.9 \mathrm{~mm}$ on its side $(\theta=$ $\pi / 2)$ and $\xi=9.9 \mathrm{~mm}$ in its rear $(\theta=\pi)$.

Since the Faraday threshold $\Gamma_{F}$, the height of liquid above the barrier $H_{b}$ and the droplet diameter $2 R$ were difficult to modify from one experiment to the next, we adjusted the shaking acceleration $\Gamma$ to get a $50 \%$ transmission for both experiment. We report the exact values of these four parameters $\left(\Gamma, \Gamma_{F}, R, H_{b}\right)$ for the two different experiments when mentioned. Other parameters were unchanged.

Data were acquired with a high-speed camera (Photron $S A 5$ monochrome) at $4000 \mathrm{~Hz}$. A $105 \mathrm{~mm}$ lens and a teleconverter were mounted on the camera. The image size was $1000 \times 1024$ pixels, each pixel imaging a square of side length $31 \mu \mathrm{m}$. The lighting and the camera view were both from the side and top by virtue of a semi-reflective mirror, as in the experimental arrangement described in Ref. [24]. The powerful LED lights were switched on a few seconds before the image acquisition started, then switched off at the end of the recording. The lighting time of each event was thus less than $30 \pm 3 \mathrm{~s}$, which limited heating of the bath. The room temperature was measured after each recorded trial.
Images were processed as follows. (i) The time at which the amplitude of Faraday waves was minimum was deduced from the standard deviation of the distribution of pixel gray levels. This time defined the reference clock. (ii) A low precision detection of the droplet was performed with image gradient and image thresholding tools. (iii) We detected the radius of the crater formed by the impacting droplet by means of circle detection tools. The radius of the crater increased linearly with time directly after each impact. This time evolution was fitted to determine the exact impact time, as in Ref. [24]. The impact phase corresponds to the phase at which the droplet lands on the bath regarding the reference clock at a frequency $\Omega / 4 \pi=40 \mathrm{~Hz}$. (iv) Finally, we detected the impact point with high accuracy using a pattern matching technique.

\section{OBSERVATIONS}

\section{A. Crossing probability}

Eddi et al. [19] did their experiment with several different droplets to vary the walker speed. For the sake of reducing variability between successive crossing attempts, we worked with only one droplet. We changed the incoming speed of the walker by tuning $\Gamma$. This preliminary experiment was made with $H_{b}=1.17 \pm 0.025 \mathrm{~mm}, \Gamma_{F}=4.37 \mathrm{~g}$ and a droplet of diameter of $2 R=750 \mu \mathrm{m}$. The ratio of the number of crossing events to the number of crossing attempts defines the crossing probability reported in Fig. 3. For the geometry and drop size considered, these experimental precautions limited the transition range for unpredictable crossing of the barrier to between $3.94 \mathrm{~g}$ (where everything was reflected) and 4.01 $g$ (where everything was transmitted) corresponding respectively to droplet velocities of 11.8 and $12.2 \mathrm{~mm} / \mathrm{s}$.

Our observations confirmed the "unpredictable tunneling" observed by Eddi [19], but the range of walking speeds was strongly reduced from around $4 \mathrm{~mm} / \mathrm{s}$ in the Eddi's experiment to $0.4 \mathrm{~mm} / \mathrm{s}$ here. The reduction of the variability in the initial conditions led to a smaller transition range, confirming that such variability played a large role in the crossing probability reported in the experiments of Eddi et al. [19]. In the following sections, we will show that our experiment is sufficiently well-controlled to distinguish if "unpredictability" comes from the variability of external parameters or if it is an intrinsic feature of walkers.

\section{B. Walker trajectory at $50 \%$ crossing probability}

To explore the nature of walker tunneling, we measured the trajectories of walkers incoming on a barrier with a crossing probability of $50 \%$ as arose with $\Gamma=3.81 \mathrm{~g} \pm 0.002 \mathrm{~g}$, $H_{b}=1.24 \pm 0.025 \mathrm{~mm}, \Gamma_{F}=4.426 \mathrm{~g}$, and droplet diameter $2 R=840 \mu \mathrm{m}$. The same droplet was sent 49 times toward the barrier. Figure 4 shows the superposition of the 49 launches and indicates the high reproducibility of the launching process. Specifically, the beam formed by the incident trajectories had a width of $40 \mu \mathrm{m}$ and an incident angle $\theta_{i}$ relative to the normal to the barrier of $0.8^{\circ} \pm 0.1^{\circ}$. The high-speed camera recorded the walker's impact position and time with a precision of $3 \mu \mathrm{m}$ and $0.25 \mathrm{~ms}$, respectively.

Far from the barrier, reflected and transmitted trajectories are not discernible as they are completely mixed in the $(x, y)$ 


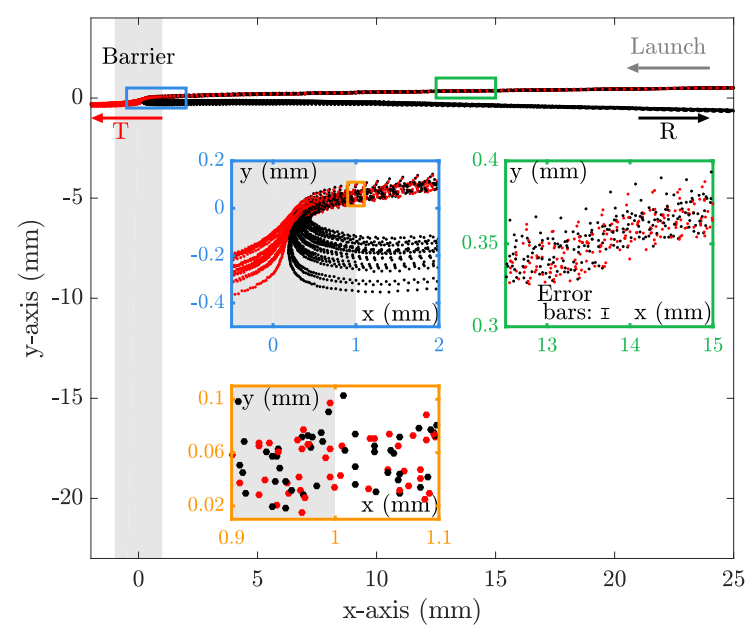

(a)

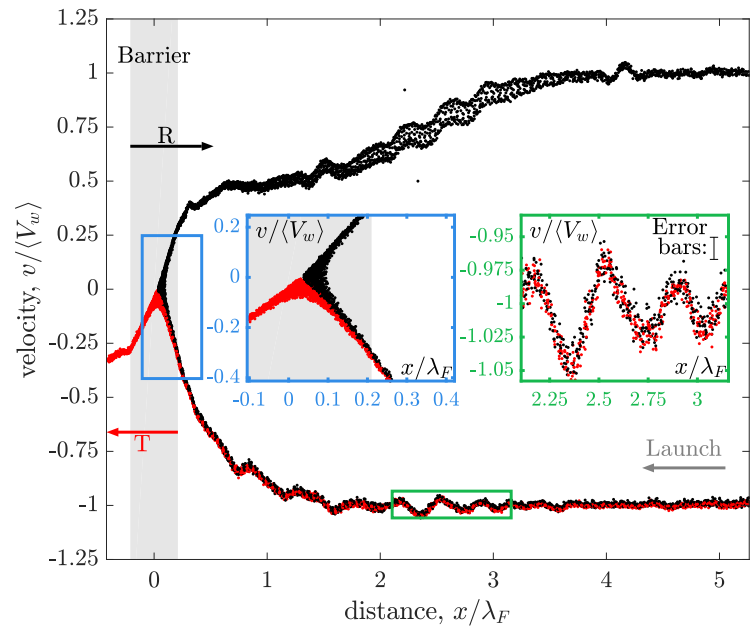

(b)

FIG. 4. (a) Trajectories in physical space. The reflected trajectories are in black, the transmitted trajectories in red. Points indicate the location of each impact between the droplet and the liquid surface. Green inset: close-up view of the beam of trajectories far from the barrier. The half-width of the beam is $20 \mu \mathrm{m}$. Blue inset: close-up view of the trajectories above the barrier. Orange inset: close-up view of the impact positions at the barrier rim. (b) Trajectories of walkers in the position-velocity phase space. Green inset: close-up on velocity fluctuations. Blue inset: close-up on the separation point.

space [see Fig. 4(a), green inset]. Trajectories are still indiscernible when stepping onto the barrier (orange inset). The trajectories remain indiscernible until $x=0.05 \lambda_{F}(0.25 \mathrm{~mm})$ just before the center of the barrier, where they separate (blue inset).

A typical representation of the system in the positionvelocity phase space is shown in Fig. 4(b). Sufficiently far from the barrier $\left(x>3 \lambda_{F} \sim 15 \mathrm{~mm}\right)$, the trajectories form a beam. The approach speed is constant with no fluctuation. We define the free walking speed $V_{w}$ as the mean speed of the walker in the range $3 \lambda_{F}<x<5 \lambda_{F}$. At a distance of $x \sim 3 \lambda_{F}$, the walker speed starts fluctuating in a repeatable manner. For $x<2 \lambda_{F}$, it decreases and some fluctuations persist. Finally, the speed almost vanishes when the droplet reaches the barrier's midpoint [Fig. 4(b), blue inset]. The beam trajectory cleanly separates into reflected (black) and transmitted (red) trajectories before stepping on the barrier. We note that during the approach to the barrier the trajectories intersect each other in the position-velocity phase space (Fig. 5). This provides clear evidence of the undercharacterization of the system by position and velocity only, since the horizontal dynamics of walkers is known to be non-Markovian and their vertical dy-

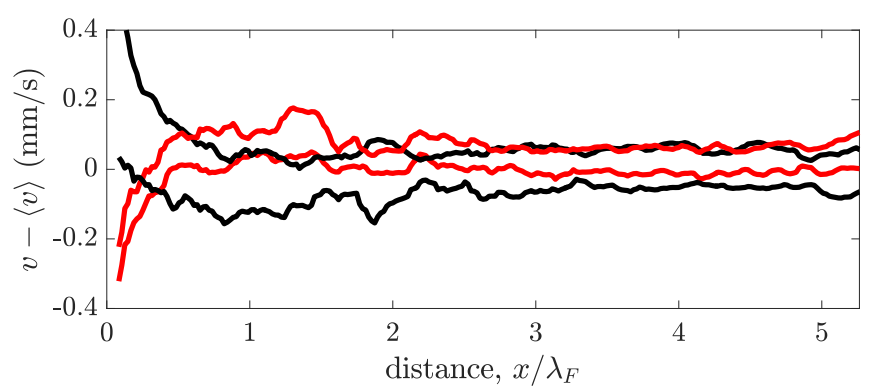

FIG. 5. Four trajectories in the position-velocity phase space. The ensemble speed average $\langle v\rangle$ has been subtracted from the original data to deduce speed fluctuations, $v-\langle v\rangle$. namics is not taken into account. Furthermore, some walkers that are faster than the ensemble mean $\langle v\rangle(x)$ are reflected whereas some that are slower are transmitted, indicating the shortcomings of classical mechanics of pointlike particles in describing the tunneling behavior when the pilot-wave field is not considered.

\section{Long-range effect of submerged boundaries}

The repeatable fluctuations of the walker speed starting at about $3 \lambda_{F}$ [see Fig. 4(b)] may be attributed to the influence of the barrier on the waves. Indeed, the attenuation factor of a wave perfectly reflected by a barrier at a distance $x$ is approximately $\exp (-2 x / \xi)$, where $\xi=1.7 \lambda_{F} \sim 8.1 \mathrm{~mm}$ is the wave damping length in front of the walker. Assuming that the walking speed $v$ is proportional to the wave amplitude, one expects the speed variations relative to the free walking speed $V_{w}$ also to be of the order of $\exp (-2 x / \xi)$. At $x=$ $3 \lambda_{F}$, we expect these relative fluctuations to be of the order of $\pm 2.5 \%$. This order of magnitude is comparable to that observed $\sim \pm 0.25 \mathrm{~mm} / \mathrm{s}$, corresponding to $2.1 \%$ of the free walking speed $V_{w}$ [see Fig. 4(b), green inset]. Therefore, these fluctuations reflect the wave-mediated nonlocal influence of the barrier on the walker.

For the 49 launches with the same droplet, we observed some slight differences. The distribution of free walking speed $V_{w}$ is reported in Fig. 6. It has a standard deviation $\sigma\left[V_{w}\right]$ of $150 \mu \mathrm{m} / \mathrm{s}$ corresponding to $1 \%$ of the ensemble mean walking speed $\left\langle V_{w}\right\rangle$. We observed that these variations are actually correlated with small variations of impact phase (Fig. 7).

Actually, droplets were launched from a channel of halfwidth $\lambda_{F}$, which is smaller than the damping length $\xi$. Therefore, the effect of such initial confinement was very strong (the damping length of the walker waves in free space is greater than $1.7 \lambda_{F}$ ) and it may have changed the droplet vertical dynamics. We observed that between different trials, the walker was launched with slightly different impact phases 


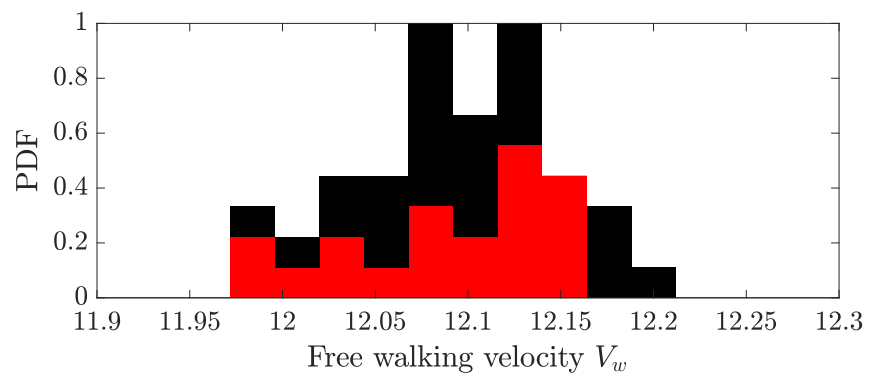

FIG. 6. Distribution of free walking velocities $V_{w}$. Red: Transmitted trajectories. Black: reflected trajectories.

$\phi_{w}$. These differences persisted as the droplet approached the barrier (Fig. 8). Droplets bouncing with different phases impact a wave with a different slope. Thus, they receive a different horizontal kick and they move at a different speed $V_{w}$. The variations of free walking speed $V_{w}$ are thus most likely associated with the preservation of the impact phase $\phi_{w}$ inherited from the interaction with the launchers.

\section{Oscillations of the probability density function}

The probability density function (PDF) is defined as the time spent by walkers of the experiment at a given position. In several settings, including corrals $[13,14]$ and a recent analogy of Friedel oscillations [29], correlations between droplet speed and position have led to the emergence of coherent, quantumlike statistical patterns. We briefly explore such a possibility in walker tunneling. The PDF of incoming and reflected walkers is plotted in Fig. 9, for the range $x \in$ $\left[2 \lambda_{F}, 3 \lambda_{F}\right]$ in which the speed oscillates without decreasing significantly. The constant component of the PDF, denoted $\mathrm{PDF}_{w}$, is removed with a high-pass filter. PDFs of both incoming and reflected walkers show oscillations with wavelength

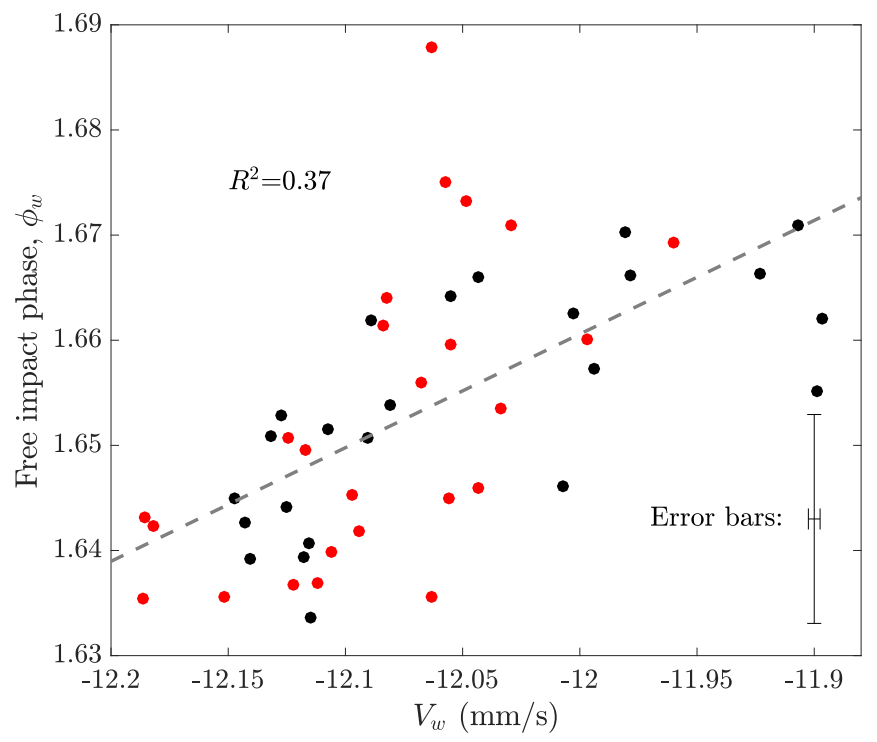

FIG. 7. Positive correlation between the free walking speed $V_{w}$ and the free impact phase $\phi_{w}$. Both quantities are averaged from instantaneous quantities, $v$ and $\phi$, for impact distances in the range $3 \lambda_{F}<x<5 \lambda_{F}$.

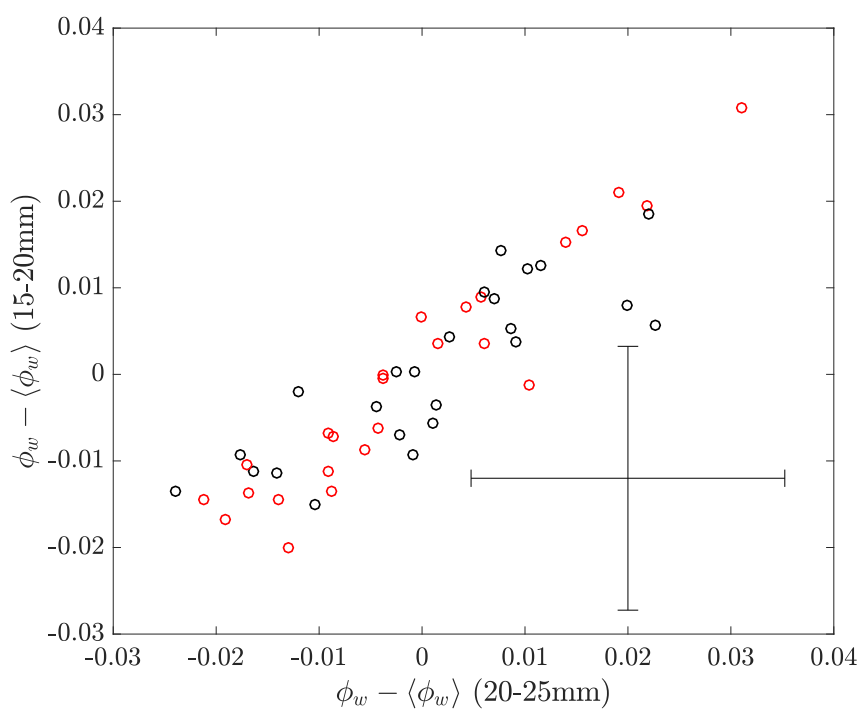

FIG. 8. Deviation of the impact phase from its ensemble average in the interval $15<x<20 \mathrm{~mm}$ from the barrier center $(x=0)$ plotted against the same deviation in the interval $20<x<25 \mathrm{~mm}$.

of approximately $\lambda / 3$ that are phase-shifted by half a period. This phase shift is consistent with that arising for reflected waves, and acts to suppress a coherent statistical signature of the form reported elsewhere [13,14,29]. Droplet speed variations for transmitted trajectories could not be computed because transmitted trajectories were beyond the field of view.

\section{E. Motion in the lateral direction}

The incident angles $\theta_{i}$ are reported in the inset of Fig. 10(b). As noted previously, the walker was launched onto the barrier with a slight incident angle $0.81^{\circ} \pm 0.1^{\circ}$ owing to technical difficulties associated with making perfect normal launches.

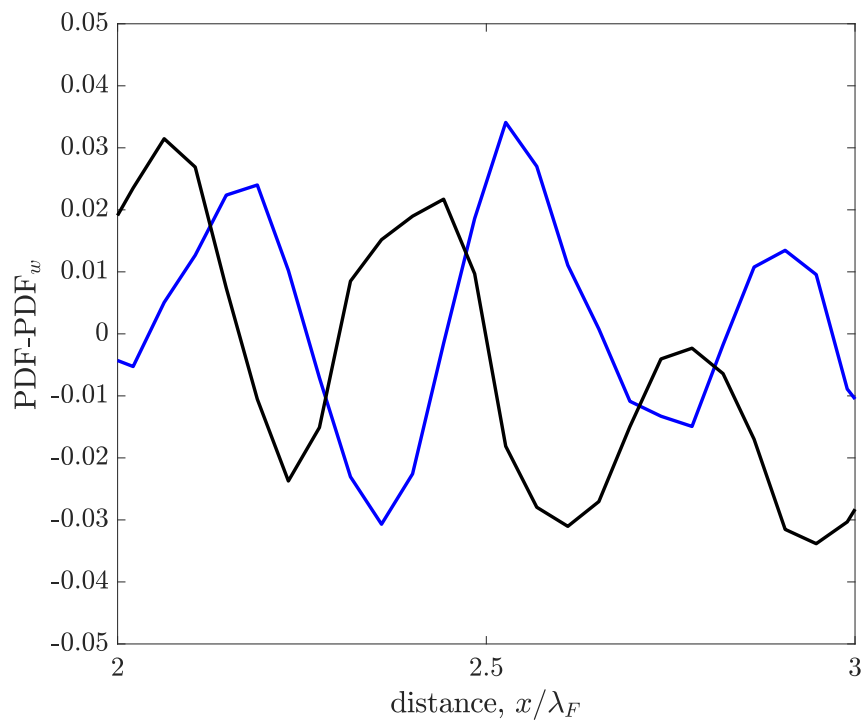

FIG. 9. High-pass filtered probability density function $\left(\mathrm{PDF}_{\mathrm{PDF}}\right)$ of incoming and outgoing walkers resulting from their velocity fluctuations. Blue: incoming trajectories. Black: outgoing reflected trajectories. 


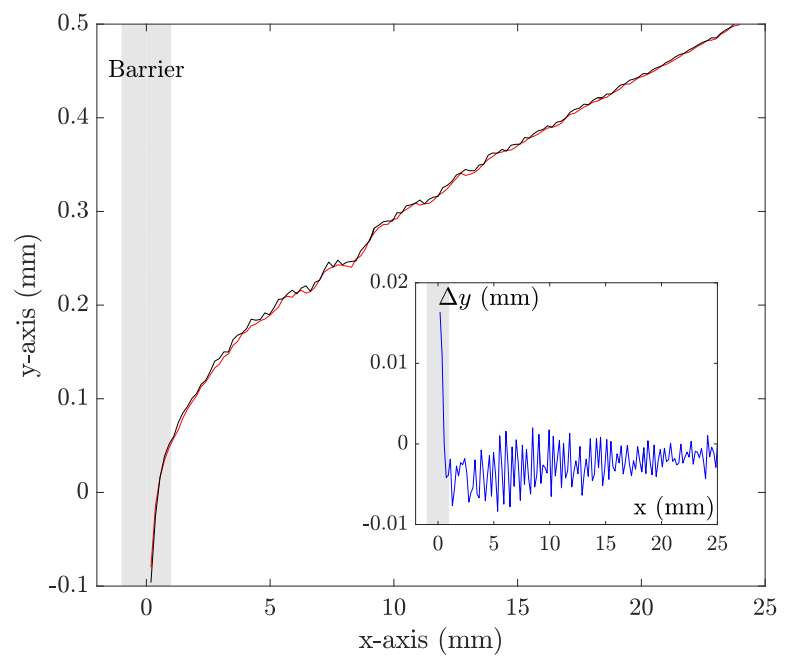

(a)

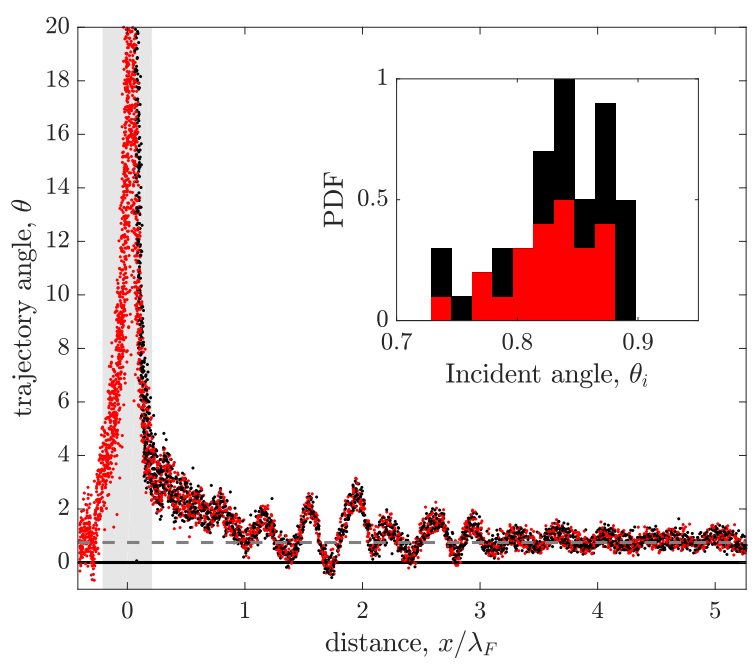

(b)

FIG. 10. (a) Evolution of the mean $y$ position of transmitted (red) and reflected (black) trajectories. Inset: plot of the difference of mean $y$ position as a function of distance to the barrier. (b) Evolution of the trajectory angle $\theta$. The gray dashed line corresponds to the mean incident angle $\theta_{i}$ far from the barrier. Inset: Distribution of incident angles. Red: Transmitted trajectories. Black: reflected trajectories.

This small angle exists because the walker has a small velocity component in the $y$ direction $V_{y} \ll V_{x}$. The average $y$ position of all transmitted and reflected trajectories is represented as a function of the distance $x$ to the barrier in Fig. 10(a). No shift is directly observed, which suggests that the switch between reflection and transmission is not directly related to $\theta$. Now considering the difference of the mean $y$ position of the transmitted trajectories with the mean $y$ position of the reflected trajectories [inset Fig. 10(a)], we observe that the separation occurs at $0.45 \mathrm{~mm}$ from the barrier center, so already above the barrier.

Before the walker reaches the barrier, the trajectory angle $\theta$ also slightly varies along the walker trajectory due to the waves interaction with the barrier [Fig. 10(b)]. When the walker arrives close to the separation point at $0.25 \mathrm{~mm}$ from the barrier center, it moves momentarily along the barrier (a)

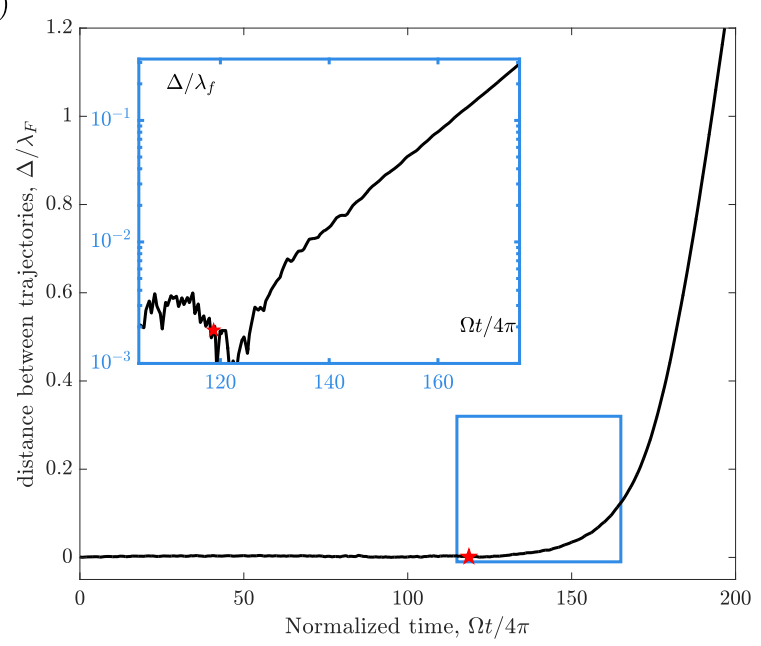

(b) $\Omega t / 4 \pi=10 \quad \Omega t / 4 \pi=140 \quad \Omega t / 4 \pi=170$

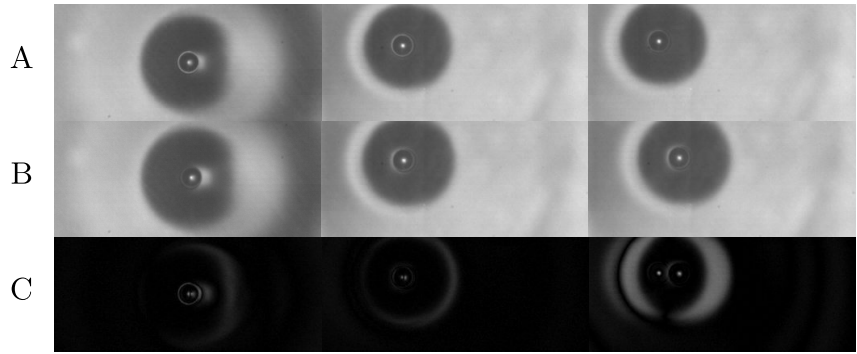

FIG. 11. (a) Exponential divergence of the physical distance between two initially close trajectories with different outputs $\left(V_{w}=\right.$ $12.196 \mathrm{~mm} / \mathrm{s}$ with initial speed difference about $0.1 \%$ ). Inset: lin$\log$ representation of the divergence. The slope corresponds to the Lyapunov exponent. Red star $(*)$ corresponds to the point at which the walker steps onto the barrier. (b) A and B: gray images of the wave field 1 and 2 far from the barrier, at the beginning and after the separation. C: Differences of intensity between images of the wave field 1 and 2.

$\theta \simeq 90^{\circ}$. After the walker is either transmitted or reflected, $\theta$ is again reduced in amplitude.

The small incident angle slightly increases the distance the walker has to travel above the barrier which would correspond to an increase of the perceived barrier width of $\Delta w / w=$ $10^{-4}$. Furthermore, the effect of incident angles on the penetration depth of walker has been observed by Pucci et al. [30]. They showed that when the incident angle is increased, so is the probability to be reflected by a barrier. Thus, a small deviation on incident angle could slightly increase the critical speed required to cross the barrier.

\section{F. Divergence of trajectories above the barrier}

The separation distance $\Delta(t)=$ $\sqrt{\left[x_{1}(t)-x_{2}(t)\right]^{2}+\left[y_{1}(t)-y_{2}(t)\right]^{2}}$ between two similar trajectories 1 and 2 with free walking speed $V_{w}=$ $12.196 \pm 0.013 \mathrm{~mm} / \mathrm{s}$ is plotted in Fig. 11(a). This distance is almost constant far from the barrier and increases exponentially on the barrier as seen in a lin-log plot [inset Fig. 11(a)]. The slope corresponds to the Lyapunov exponent. This divergence is also strongly marked on the 
wave fields, Fig. 11(b). While this exponential divergence is not necessarily a proof of chaos, it at least suggests that the barrier acts as a saddle point in the phase space of the walker.

\section{ANALYSIS OF THE UNPREDICTABILITY}

\section{A. Distinguishing between possible causes of unpredictability}

Our experiment, although better controlled than previously reported tunneling experiments [19], remains unpredictable. The small variations of initial parameters lead to markedly different outcomes: transmission or reflection. Three different physical paths may lead to this behavior for a dynamic system (i) First, in classical point mechanics, the barrier is seen as a saddle point that reflects low-velocity trajectories and transmits high-velocity trajectories. The unpredictability may then come from an insufficient knowledge of the initial conditions [Fig. 12(a)]. For instance, if the initial velocity is known within uncertainty bounds (red-black striped initial box) that overlap the single manifold separating reflected and transmitted trajectories, then the barrier crossing cannot be predicted. (ii) Second, some variability may also come from external noise during the experiment (such as ambient air currents) which may switch the particle trajectory from one side of the manifold to the other [Fig. 12(b)]. (iii) The path to unpredictability is chaos: any small change in the initial conditions may give different outputs. The difference with a simple saddle-point is that owing to stretching and folding, the phase space of a chaotic system appears fractioned into a myriad of small basins of attraction for each output. Even if we know the initial conditions extremely well, the lack of knowledge of their exact value may yield "unpredictable" results. Transmitted and reflected trajectories then appear mixed at a scale larger than the measurement uncertainty (small red stripped box); they then sort out at the foot of the barrier [Fig. 12(c)].

To distinguish between these possible sources of apparent unpredictability, we first review the magnitude of experimental variability and precision measurement. Second, we analyze the intrinsic variability of walkers impinging on a submerged barrier.

\section{B. External sources of variability}

As the problem of crossing the barrier may be subjected to small uncontrollable changes from external parameters, for instance the change of free walking speed, we focused on (i) the precision of our measurements and (ii) the quantification of uncontrollable fluctuations that may influence the experiment.

The uncertainty on the initial walking velocity can be decreased until $\Delta V_{w} / V_{w}<0.03 \%$ by considering several impacts (see Appendix A). We thus reject scenario (i) since initial walking velocity $V_{w}$ is known with a good accuracy compared to the group dispersion of walking speeds $\simeq 1 \%$, Fig. 6(a).

Figure 5 has already shown that scenario (ii) is unlikely, since the initial speed difference between incoming walkers seems to be preserved until they get very close to the barrier. Moreover, we analyzed the speed fluctuations around the instantaneous group speed $(v-\langle v\rangle) /\left\langle V_{w}\right\rangle$ in the constant

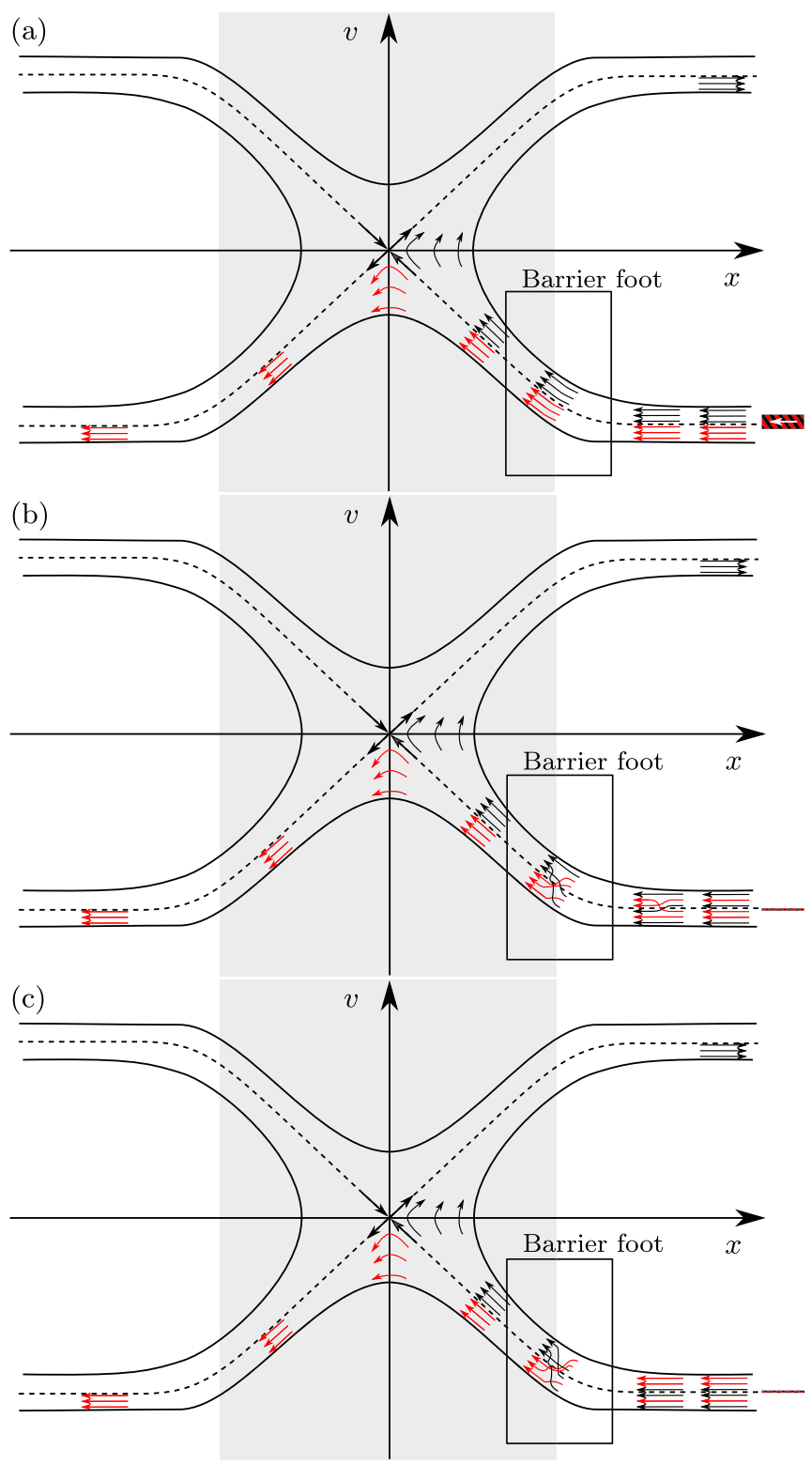

FIG. 12. Schematic of causes of unpredictability in the positionvelocity phase-space. (a) Large uncertainties on the initial conditions for a classical saddle-node bifurcation. (b) Noise-induced variations before and above the barrier. (c) Chaos-induced unpredictability resulting from fractal basins of attraction. Additional dimensions of the phase space could be associated with bouncing phase, for instance.

velocity region $\left(5 \lambda_{f}>x>3 \lambda_{F}\right)$ for each trajectory. These fluctuations have a standard deviation of $\sigma\left[(v-\langle v\rangle) /\left\langle V_{w}\right\rangle\right]=$ $0.09 \%$, much smaller than $\sigma\left(V_{w}\right)=1 \%$ of the total speed range of the group. The noise level in our experiment was thus insufficient to force trajectories to randomly cross the manifold during the approach to the barrier. We can thus reject the noise-induced unpredictability, scenario (ii), which was avoided here because the bath was protected from air currents with a lid. Although no signal appears in the distribution of velocity variations, some other possible external sources of variability are analyzed in Appendix B. Among them, temperature variations of the bath could significantly modify 


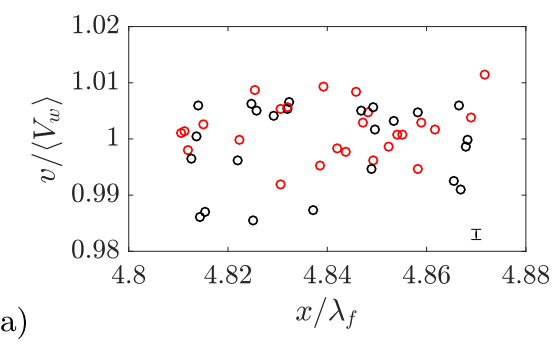

(b)

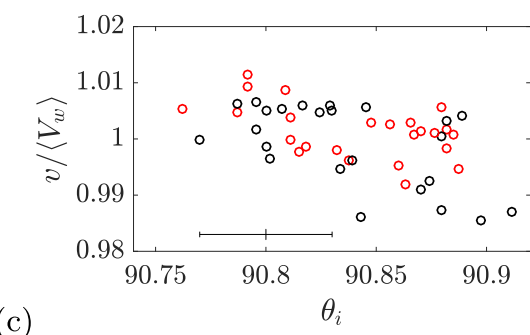

(c)

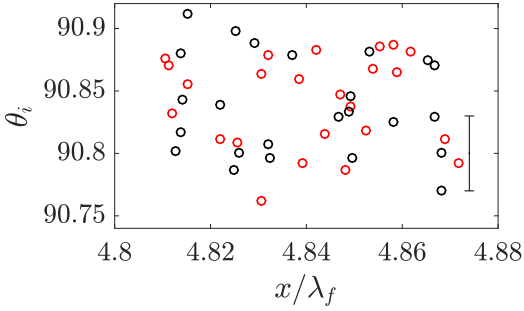

FIG. 13. Cross sections of the phase space of a walker at a distance of $23 \mathrm{~mm}\left(4.84 \lambda_{F}\right)$ from the barrier. Speeds and angles were averaged over 11 successive impacts. (a) Position vs speed. (b) Position vs trajectory-angle. (c) Velocity vs trajectory-angle.

the Faraday threshold, and subsequently the walker dynamics. Although these variations have not been directly measured, we present theoretical arguments in Appendix B that suggest that they were limited and did not likely modify the walker dynamics in our experiments.

\section{Analysis of intrinsic variability}

\section{Trajectories in the phase space}

As the measurement error and the sources of noise are now quantified, we can proceed with a deeper analysis of the basins of attraction of reflected and transmitted trajectories.

The phase space to consider consists of the variables that describe the incoming walker in the free walking zone, namely the walker speed $v$, the trajectory angle $\theta$, and the bouncing position $x$. Since the impact phase $\phi$ is correlated to the walker speed $v$ in the free walking zone, we do not consider it to be an independent dimension of the phase space. Similarly, the phase space does not include any specific dimension associated with the waves. Indeed, as the damping time of the waves is $0.14 \mathrm{~s}$ and the damping length $8.8 \mathrm{~mm}$, waves either emitted during previous passages of the walker (several seconds before) or reflected from the boundaries (several centimeters away) are fully damped in the free walking zone. The wave field is therefore entirely prescribed by $(x, v, \theta)$.

The three two-dimensional cross-sections of this threedimensional phase space are represented in Fig. 13, for incoming walkers at a distance $x \sim 4.8 \lambda_{F}$ from the barrier. At this distance, distributions of reflected and transmitted trajectories appear to be fully mixed. Again, the region in which they overlap is much wider than the measurement error bars, confirming that scenario (i) of Sec. IV must be ruled out.

To confirm scenario (iii), we try to determine the distance from the barrier at which the distributions of reflected and transmitted trajectories become distinguishable (i.e., unmixed) in the phase space $(x, v, \theta)$ of the incoming walker. This discernability is quantified with the $p$-value of a twosample $t$-test, which represents the probability that the observed difference between distributions is due to chance. We first perform this test in each dimension of the phase space separately, at different distances $x$ from the barrier [Fig. 14(a)]. We consider that distributions are distinguishable (i.e., reflected and transmitted trajectories are separated) when $p<2 \%$, and we define the demix distance $D$ as the minimum distance from the barrier center below which $p$ is systematically less than $2 \%$. For example, the demix distance based on speed measurements is $D_{v}=0.23 \lambda_{F}$. In other words, one could predict if a walker would be reflected or transmitted based on one measurement of its speed $v$ only if this measurement is performed when the walker is already on the barrier, at $x<D_{v}=0.23 \lambda_{F}$. The demix distances associated with $x$ and $\theta$ are $D_{x}=0$ and $D_{\theta}=0.2 \lambda_{F}$, so $x$ and $\theta$, considered separately, are worse predictors of the walker's outcome.

We may expect that measuring more than one variable among $(x, v, \theta)$ would provide more information, and possibly allow one to predict the outcome earlier. This intuition is illustrated with the $(x, v)$ cross-section at $x \simeq 0.22 \lambda_{F}$ from the barrier center, represented in Fig. 14(b). Clearly, the exact

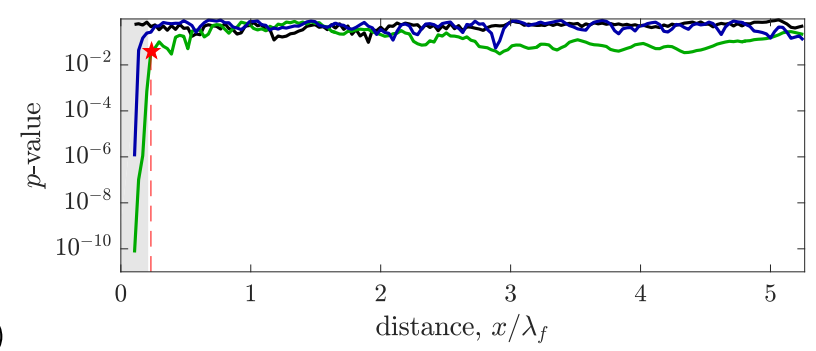

(a)

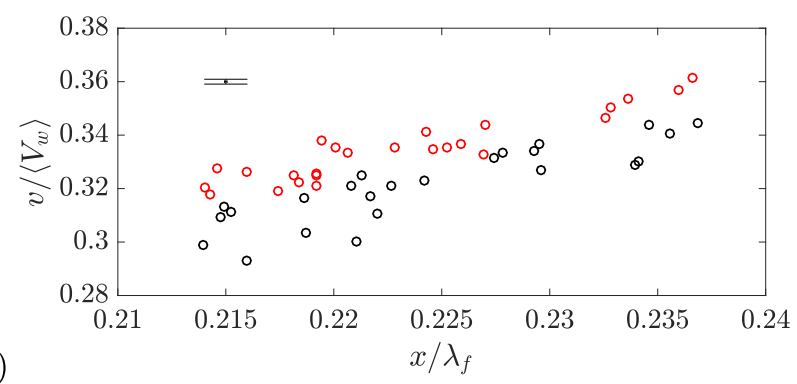

(b)

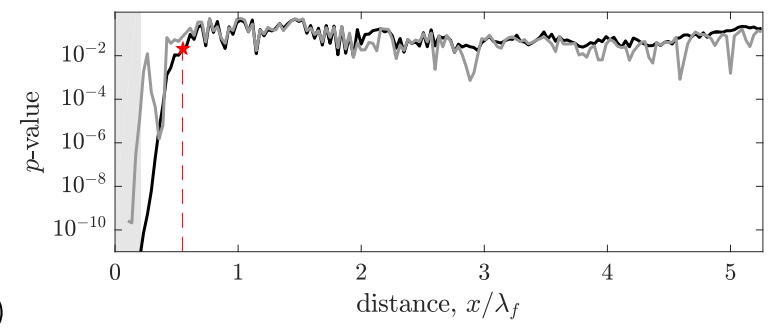

FIG. 14. (a) $p$-value of two-sample $t$-tests to distinguish reflected and transmitted walker trajectories. Green: $p$-value computed with the velocities $v$. Blue: $p$-value computed with the trajectory-angles $\theta$. Black: $p$-value computed with the impact positions. (b) Impact position-Velocity cross section of the phase space of the crossing of the barrier by a walker at a distance of $1 \mathrm{~mm}\left(0.22 \lambda_{F}\right)$. (c) $p$-value of the PCA analysis in the $(x, v)$ cross section (black) and in the complete $(x, v, \theta)$ space (light gray). In both panels (a) and (c), red $\operatorname{star}(*)$ denotes $D_{M}$, the demix distance. 
TABLE II. Demix distances $D_{M}$ as a function of the phase-space cross-section considered.

\begin{tabular}{lccccccc}
\hline \hline Phase space & $x$ & $v$ & $\theta$ & $x-v$ & $x-\theta$ & $v-\theta$ & $x-v-\theta$ \\
\hline$D_{M}(\mathrm{~mm})$ & 0 & 1.1 & 0.95 & 2.6 & 0.95 & 1.1 & 1.85 \\
$D_{M} / \lambda_{F}$ & 0 & 0.23 & 0.20 & 0.55 & 0.20 & 0.23 & 0.39 \\
\hline \hline
\end{tabular}

knowledge of $x$ alone is insufficient to know if the walker will be reflected or not, since $D_{x}<0.23 \lambda_{F}$. Similarly, the exact knowledge of $v$ alone is barely sufficient to reasonably predict the outcome, since $D_{v} \simeq 0.23 \lambda_{F}$. Figure 14(b) reveals that reflected and transmitted trajectories are already well separated in the $(x, v)$ plane. However, the manifold that separates them is not uniquely defined in terms of either $x$ or $v$, but rather as some linear combination of $x$ and $v$.

More generally, at each distance to the barrier, there is a direction in the phase space that maximizes the separation of reflected and transmitted trajectories. We determined this direction with principal component analysis (PCA), then performed the two-sample $t$-test in this direction. The calculated $p$-value is represented as a function of the distance to the barrier in Fig. 14(c). The corresponding demix distance is reported in Table II, for phase spaces comprising different combinations of $x, v$, and $\theta$. The phase space $(x, v)$ yields the largest demix distance, at $0.55 \lambda_{F}$ from the barrier center (i.e., $0.3 \lambda_{F}$ from the barrier edge). Owing to the relatively large error on $\theta$, the demix distance in the full $(x, v, \theta)$ space is not larger than that in the $(x, v)$ space.

\section{The role of impact phase}

Trajectories are only sorted close to the barrier, which suggests that the barrier may act as a source of chaos. One further argument consistent with chaos involves the impact phase $\phi$ (Fig. 15). While the latter is nearly constant throughout the approach it dips at $x \simeq 0.5 \lambda_{F}$ then increases sharply above the barrier. The variation of the impact phase close to the barrier reflects a significant change in the drop's vertical dynamics, as is induced by the barrier's influence on its guiding wave.

The end of the unpredictability is simultaneous with variations of the impact phase. As the drop approaches the barrier, it responds to increased barrier-induced perturbations of its Faraday wave field.

In a previous study, we showed that changes in vertical dynamics during the interaction of two droplets may introduce chaos into the walker system [24]. In particular, we

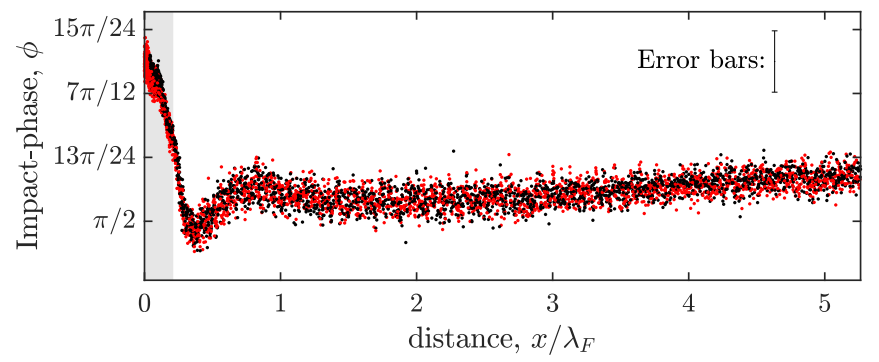

FIG. 15. Impact-phase vs distance to the barrier. Red: transmitted trajectories. Black: reflected trajectories. showed that the onset of chaos in interacting walking pairs was triggered by a change of impact phase of about $0.2 \mathrm{rad}$ which is of the same order of magnitude as the $0.12 \mathrm{rad}$ perturbation evident in Fig. 15. It thus seems likely that the walker trajectory becomes chaotic in the immediate vicinity of the barrier, which explains why its outcome cannot be predicted sooner.

\section{COMPARISON WITH QUANTUM TUNNELING}

The walker system has been considered as a hydrodynamic quantum analog although there is no doubt that the nature of walking droplet is different from that of a quantum particle $[8,9,12,13,19]$. We proceed by examining the possibility that tunneling in both systems leads to similar results in terms of distance at which the system becomes predictable.

In quantum mechanics, the particle is modeled by its statistical wave function $\Psi$. In the case of a square barrier of potential $U_{0}$ larger than the initial kinetic energy of the quantum particle $E$, the particle may tunnel with a transmission coefficient,

$$
T=\frac{4 E\left(U_{0}-E\right)}{4 E\left(U_{0}-E\right)+U_{0}^{2} \sinh \left(\sqrt{2 m\left(U_{0}-E\right)} w / \hbar\right)},
$$

where $w$ is the barrier width and $m$ the particle mass [31]. We cannot obtain any certain indication about the fate of a single particle.

In the Copenhagen interpretation of quantum mechanics, the trajectory of a quantum particle is not defined. One way to approximate the trajectory of an individual quantum particle is to perform repeated measurements on its position and momentum [32-34]. Repeated measurements have two main effects. First they relocalize the statistical wave around the last measured position of the particle, introducing thereby a pseudo locality of the waves. Second, the repeated measurements introduce significant noise due to the random projections of the wave function onto the projectors. We here perform a numerical simulation of tunneling particles for which position and momentum are repeatedly measured.

We consider a particle of wavelength $\lambda_{\mathrm{dB}}$ and momentum $2 \pi \hbar / \lambda_{\mathrm{dB}}$. We define a set of basis functions

$$
\chi_{n, l}(x)=\frac{1}{\sqrt{L}} \frac{\sin (2 \pi(x-n L) / L)}{2 \pi(x-n L) / L} e^{2 i l \pi x / L},
$$

to be used as projectors for a quantum measurement, where $L=3 \lambda_{\mathrm{dB}}$. A projection onto the state $\chi_{n, l}$ indicates that the particle is on average, after this measurement, located at the position $x=n L$ and propagates with the momentum $p=$ $l(2 \pi \hbar / L)$ with respective uncertainties $\sigma\left[x / \lambda_{\mathrm{dB}}\right]=\infty$ and $\sigma\left[p /\left(h / \lambda_{\mathrm{dB}}\right)\right]=1 /(3 \sqrt{6})$. The respective uncertainties indicate that the quantum particle may be subjected to large jumps in position but not in momentum. This set of basis functions is both orthonormal and complete on $\mathbb{R}$. For numerical reasons, $n$ and $l$ are restricted to $[-50,50]$.

We consider a quantum particle launched toward a barrier of potential $U(x)=U_{0} \exp \left(-x^{2} / w^{2}\right)$ with $w=2 L$ and $U_{0}=$ $241 \hbar^{2} / m L^{2}$. The potential $U_{0}$ matches the kinetic energy of a particle of wavelength $\lambda=L / 3.5\left(E=2 \pi^{2} \hbar^{2} / m \lambda^{2}\right)$ to ensure almost equal probabilities of reflection and transmission when the particles are launched with momentum $p=$ 


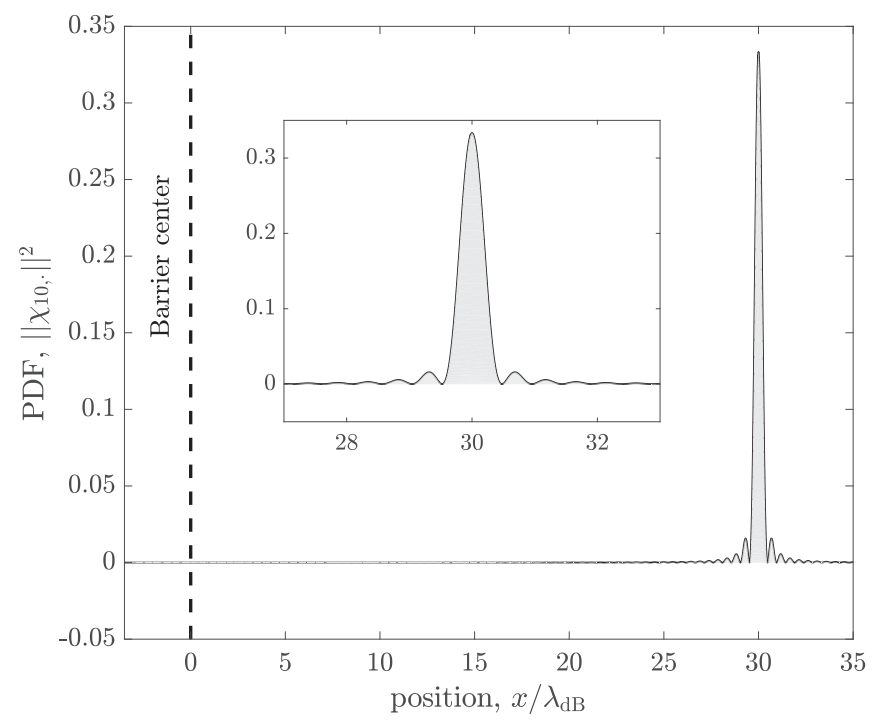

FIG. 16. Spatial probability density function of the initial states of the quantum simulation, $\Psi(0, x)=\chi_{10, .}(x)$. Inset: zoom on the projector around its mean value.

$-6 \pi \hbar / L$. We assume that the first detection takes place on the projector of $\Psi(0, x)=\chi_{n_{0}, l_{0}}(x)$ with $n_{0}=10$ and $l_{0}=$ -3 , which prescribes the initial conditions of the numerical experiment (Fig. 16). The wave packet evolves according to the Schrödinger equation,

$$
i \hbar \frac{\partial \Psi}{\partial t}=-\frac{\hbar^{2}}{2 m} \frac{\partial^{2} \Psi}{\partial x^{2}}+U(x) \Psi(x, t) .
$$

After a time interval $\tau=0.05 L^{2} \mathrm{~m} / \hbar$, the particle is measured. This time interval is chosen so that the particle moves by an average distance of $\tau p / m=0.94 L$ which roughly corresponds to the distance between two consecutive projectors.

In the simulation, the measurement consists of projecting the wave function $\Psi\left(x, t+\tau^{-}\right)$onto the above measurement basis. In perfect accordance with quantum theory, the outcome $\Psi\left(x, t+\tau^{+}\right)=\chi_{n, l}$ of this randomly simulated measurement process is obtained with the probability

$$
P_{n, l}=\left|\int_{-\infty}^{\infty} \chi_{n, l}^{*}(x) \Psi(x, t+\tau) d x\right|^{2} .
$$

The wave function $\Psi\left(x, t+\tau^{-}\right)$instantaneously collapses onto a single projector state $\Psi\left(x, t+\tau^{+}\right)=\chi_{n_{1}, l_{1}}(x)$. To define a quantum trajectory, this process is repeated 20 times to get $\Psi\left(x, t+20 \tau^{+}\right)=\chi_{n_{20}, l_{20}}(x)$.

With this procedure, we simulated 1000 trajectories as shown in Fig. 17(a). The trajectories start from a pure $\chi_{n_{0}, l_{0}}$ state corresponding to the first measurement done on a Gaussian wave packet. The trajectories separate above the barrier, in a manner reminiscent of walkers in Fig. 17(a). As we did for walkers, we compute the $p$-value of the two-sample $t$-test between the two distributions of transmitted and reflected trajectories for each position. The $p$-value is displayed in Fig. 17(b).

We determine the demix distance $D_{M}$ as the distance from the barrier center where $p<2 \%$. We repeated this numeri-

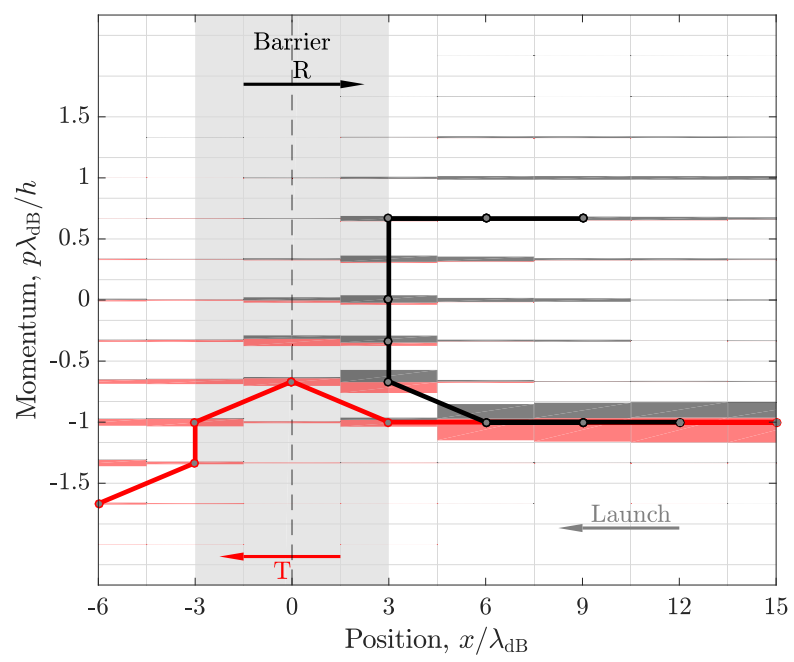

(a)

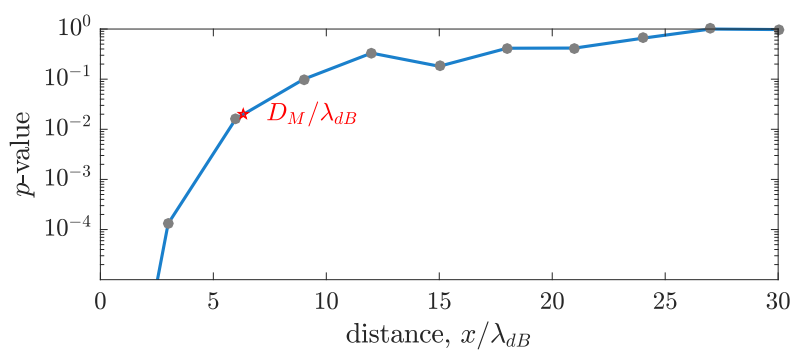

(b)

FIG. 17. (a) Trajectories of quantum particles in the positionmomentum phase space. Red: transmitted trajectories. Black: reflected trajectories. Each box corresponds to a specific projector $\chi_{n, l}$. The total number of detected trajectories is represented by the width of the beam in the box. The reflected/transmitted ratio is represented by the relative magnitude in black and red in the box. The box dimensions indicate the difference between the position and momentum mean values of two consecutive detectors. (b) $p$-value computed on distributions of transmitted and reflected trajectories of quantum particles obtained by numerical simulation. Red star $(*)$ indicates the demix distance $D_{M}$.

cal simulation with variable barrier width $w$ and found that $D_{M} \sim w$ (see Fig. 18). This result is in accordance with what we obtained for walkers, although observations could not be made for the same ratio of barrier width to wavelength. This similarity is not trivial, since the demix distance could have

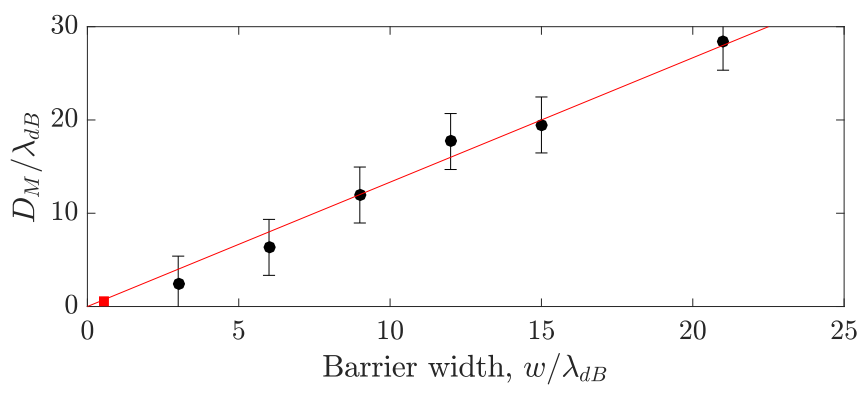

FIG. 18. Demix distance as a function of barrier width, as computed from our quantum simulations. Red square: walker experiments for which we nondimensionalize by $\lambda_{F}$ rather than $\lambda_{\mathrm{dB}}$. 
instead scaled with the other main characteristic length scale, namely, the de Broglie wavelength.

\section{DISCUSSION}

Since the first work of Eddi et al. [19], two physical phenomena were invoked to explain the "unpredictability" of walker tunneling, namely, the uncertainty of initial conditions [21] or the peculiar build up of the wavefield [20]. With our precise experiments, we showed that the uncertainty in the initial condition and the noise of the experiment cannot be held responsible for the "unpredictable tunneling" of the walkers. Rather, our observations are consistent with chaos. We observe that trajectories sort out at a short distance before stepping on the barrier. At this distance an exponential divergence between nearby trajectories is observed. Also at this distance, the interaction of the waves with the barrier is sufficient to influence the drop's vertical dynamics, alterations in which are known to be a source of chaos [24].

Walker and quantum tunneling exhibit several similar features. Both are unpredictable in the sense that the outcome cannot be directly deduced from the initial kinetic energy of the particle. Both are also unpredictable in the positionvelocity cross section of the phase space. The variation of the tunneling probability with physical parameters observed in the walking droplets is also a key element of nuclear decay at atoms as well as of other quantum tunneling processes, e.g., with ultracold atoms [35]. This similarity suggests that a classical nonlinear theory may be built to account for tunneling similarly as quantum mechanics does. This possibility has been proposed by Shudo and Ikeda [36] in the context of semi-classical theory of tunneling (see Ref. [37] for a more recent overview on this topic).

The repeated measurements done on a tunneling quantum particle allow one to approximate the particle trajectory to make comparisons with walkers. These repeated measurements make the quantum particle partially classical. The statistical analysis of these trajectories shows that the unpredictability vanishes only at a relatively short distance from the barrier. However, the nature of the theoretical descriptions of the tunneling in the two systems is markedly different. For walkers, the unpredictability results from chaos generated by the subtle coupling between the waves and the particle. Conversely, tunneling of quantum particles is described by the wave function and measurements introduce probabilistic results prescribed by the squared amplitude of the wave function.

Our study provides further evidence that a classical particle-wave association may exhibit statistical behavior reminiscent of a quantum system. Specifically, the walker system demonstrates that "unpredictable" tunneling is not a purely quantum oddity since it can be achieved with a classical system. The origins of the loss of predictive power in the walker system does not rely on the intrinsic variability of the experiment but is most likely rooted in the fast time scale associated with the drop's vertical dynamics. For a quantum particle, unpredictability is ensured by the paradigm: The particle is described entirely by a statistical wave. No fast timescale is involved in the standard description of a quantum particle, for which the origins of unpredictability, if any, remain unknown.

\section{ACKNOWLEDGMENTS}

Authors warmly thank Jim Bales for his valuable advice and for granting access to the high-speed imaging facility of the Edgerton Center (MIT). This work was financially supported by the FNRS Grant No. CHAR. RECH.-1.B423.18 (Tadrist L.). J.B. gratefully acknowledges the support of the NSF through Grant No. CMMI-1727565. L.T., J.B., and T.G. designed the walker tunneling experiment. L.T. did data acquisition and data analysis. P.S. performed the numerical experiment of quantum tunneling. L.T., J.B., and T.G. wrote the manuscript. Authors declare no competing interest.

\section{APPENDIX A: MEASUREMENT ERRORS}

The accuracy of impact location determined by pattern matching is about $1 / 10$ of a pixel (given by the Matlab least-squares fitting algorithm). We thus obtain an absolute error on position $|\Delta x|=3 \mu \mathrm{m}$, which corresponds to $1 \%$ of the interstep distance $b \simeq 4 \pi V_{w} / \Omega$ associated with the free walking far from the barrier.

The error on impact time is $\left|\Delta t_{i}\right|<0.25 \mathrm{~ms}$ (the recording rate). This represents a measurement uncertainty on the impact phase relative to the Faraday period of $|\Delta \phi| / 2 \pi<1 \%$. The uncertainty on the instantaneous speed $v$ is bounded by

$$
\frac{|\Delta v|}{v} \leqslant \frac{\Omega|\Delta x|}{4 \pi V_{w}}+\frac{|\Delta \phi|}{2 \pi}<2 \% .
$$

The free walking speed $V_{w}$ corresponds to an average over $N \sim 33$ successive rebounds, over which the instantaneous speed $v$ does not fluctuate significantly. Although $v$ has a measurement error of about $120 \mu \mathrm{m}$, the error made on the free walking speed is considerably smaller, $\Delta V_{w}=\Delta v / N \simeq$ $3 \mu \mathrm{m} / \mathrm{s}$, which corresponds to a relative error of $0.03 \%$.

\section{APPENDIX B: MAIN SOURCES OF EXTERNAL VARIABILITY}

The "unpredictability" of tunneling may come from some uncontrolled variations of external parameters. The velocity of a walking droplet mainly depends on the drop radius $R$, the shaking acceleration $\Gamma$ and the temperature $T$ through the Faraday threshold $\Gamma_{F}(T)$. In the present experiment, $\Delta R=$ 0 since the same droplet was used for all the launches. The variations of $V_{w}\left(R, \Gamma, \Gamma_{F}\right)$ are then given by

$$
\frac{\Delta V_{w}}{V_{w}} \leqslant\left|\frac{\partial V_{w}}{\partial \Gamma}\right| \frac{\Gamma}{V_{w}} \frac{\Delta \Gamma}{\Gamma}+\left|\frac{\partial V_{w}}{\partial \Gamma_{F}}\right| \frac{\Gamma_{F}}{V_{w}} \frac{\Delta \Gamma_{F}}{\Gamma_{F}} .
$$

The variation of velocity with shaking acceleration for the droplet size considered here may be found in the work of Molaček and Bush [[5], Fig. 5(a)], where $V\left(\Gamma / \Gamma_{F}\right)$ is reported:

$$
\left.\frac{\partial V_{w}}{\partial\left(\Gamma / \Gamma_{F}\right)}\right|_{\Gamma_{F}}=\Gamma_{F}\left|\frac{\partial V_{w}}{\partial \Gamma}\right|_{\Gamma_{F}} \simeq 73.5 \mathrm{~mm} / \mathrm{s}
$$

We thus find $\left|\partial V_{w} / \partial \Gamma\right| \Delta \Gamma / V_{w}=0.26 \%$. 
Some possible variations of the Faraday threshold may be due to temperature variations of the oil bath. The room temperature near the shaker was recorded throughout the $10 \mathrm{~h}$ of the experiment and was almost constant at $21.5 \pm 0.2^{\circ} \mathrm{C}$. The variations of oil density $\rho$, surface tension $\gamma$, and kinematic viscosity $v$ follow,

$$
\frac{d \rho}{\rho}=-\alpha d T, \quad \frac{d \gamma}{\gamma}=-\beta d T \text { and } \frac{d \nu}{\nu}=-\varepsilon d T,
$$

with $\alpha=1.07 \times 10^{-3} \mathrm{~K}^{-1}, \beta=6 \times 10^{-5} \mathrm{~N} \mathrm{~m}^{-1} \mathrm{~K}^{-1}$, and $\varepsilon=1.0 \times 10^{-2} \mathrm{~K}^{-1}$. From there, the error on the Faraday threshold $\Gamma_{F}$ induced by temperature variations of $\Delta T=0.2 \mathrm{~K}$ could be deduced from the expression in Ref. [[7], Eq. (2.64)],

$$
\begin{aligned}
& \Gamma_{F} \simeq \frac{\Omega^{2}}{k_{0}}\left(\frac{4 v k_{0}^{2}}{\Omega}\right)-\frac{\Omega^{2}}{2 k_{0}}\left(\frac{4 v k_{0}^{2}}{\Omega}\right)^{3 / 2}, \\
& \text { with } k_{0} \simeq\left(\frac{\Omega^{2} \rho}{4 \gamma}\right)^{1 / 3}-\frac{g}{3}\left(\frac{2 \rho}{\gamma \Omega}\right)^{2 / 3} .
\end{aligned}
$$

At the first order the relative variation of the Faraday threshold is

$$
\frac{\Delta \Gamma_{F}}{\Gamma_{F}} \leqslant \frac{\Delta v}{v}+\frac{\Delta \rho}{3 \rho}+\frac{\Delta \gamma}{3 \gamma},
$$

which is clearly led by the variation of viscosity with temperature since $\varepsilon / \alpha \gg 1$ and $\varepsilon / \beta \gg 1$. We thus deduce $\Delta \Gamma_{F} / \Gamma_{F}<$ $0.2 \%$. Using again the results of Molaček and Bush [5],

$$
\left.\frac{\partial V_{w}}{\partial\left(\Gamma / \Gamma_{F}\right)}\right|_{\Gamma}=\frac{\Gamma_{F}^{2}}{\Gamma}\left|\frac{\partial V_{w}}{\partial \Gamma_{F}}\right|_{\Gamma} \simeq 73.5 \mathrm{~mm} / \mathrm{s},
$$

we obtain a velocity variation $\left|\partial V_{w} / \partial \Gamma_{F}\right| \Delta \Gamma_{F} / V_{w}=1.0 \%$. However, the mean room temperature was kept constant throughout the day by the air conditioning system of the laboratory. No thermal fluctuation lasting more than $5 \mathrm{~min}$ was observed. Since the bath had a much larger thermal inertia than the air in the room, we did not expect significant variations of the Faraday threshold in our experiment.
Another source of error is the use of a powerful LED to light the experiment. The LED had a power of $100 \mathrm{~W}$ and lit a surface of approximately $0.25 \mathrm{~m}^{2}$ after passing through a semireflective mirror (50-50). The silicone oil is transparent to visible light and we assume that the light only warms up the underlying steel plate. Some light was reflected by the lid and the free surface of the oil, so the value of $P_{s}=200 \mathrm{~W} / \mathrm{m}^{2}$ is an upper bound for the power per unit of area that reaches the plate. If all the light energy were converted into heat, then the steel plate temperature $T_{p}$ would increase by $\Delta T_{p}=$ $P_{s} \tau / \rho C_{p} e$, where $\tau$ is the illumination time, $\rho=7000 \mathrm{~kg} / \mathrm{m}^{3}$ is the steel density, and $C_{p}=470 \mathrm{~J} / \mathrm{K} / \mathrm{kg}$ is the specific heat capacity of steel and $e=4 \mathrm{~mm}$ is the plate thickness. The illumination time $\tau$ was around $30 \mathrm{~s} \pm 3 \mathrm{~s}$ which could have led to a maximum elevation of the plate temperature $\Delta T_{p}=$ $0.9 \pm 0.09 \mathrm{~K}$. The heat diffused through the liquid from the bottom plate. The thermal diffusivity of silicone oil is $D=$ $910^{-8} \mathrm{~m}^{2} / \mathrm{s}$. Assuming that the resulting heat transfer was diffusive rather than convective, the temperature of the liquid can be estimated by $T(z)-T_{0}=T_{p}[1-\exp (-\sqrt{D \tau} / z)]$ and leads to a mean bath temperature over the height of liquid $H=6.5 \mathrm{~mm}$,

$$
\frac{\bar{T}-T_{0}}{T_{p}}=\frac{1}{H} \int_{0}^{H} 1-e^{\sqrt{D \tau} / z} d z \simeq 0.1,
$$

since $\sqrt{D \tau} / H \simeq 0.27$. We finally estimate the variation of the bath temperature between trials as $\Delta \bar{T} \simeq 0.12 T_{p}\left(\Delta T_{p} / T_{p}+\right.$ $\Delta \tau / \tau) \simeq 0.02 K$. This estimation is an upper bound of the bath temperature variation between trials. We thus only expect variations of velocity in the order of $\Delta V_{w} / V_{w}=0.1 \%$.

We also performed a $\chi^{2}$ test to check the independence of the output (reflected or transmitted) to the room temperature. The computed $p=0.16$ means that there is $16 \%$ chance that the correlation measured between temperature and tunneling is due to chance. The statistical power of the test is pwr $=0.55$. With the current data set, we cannot conclude that room temperature has a significant influence on the outcome. However, we stress that this conclusion may still be misleading owing to the relatively low statistical power due to the relatively small number of launches considered.
[1] Y. Couder, E. Fort, C.-H. Gautier, and A. Boudaoud, From Bouncing to Floating: Noncoalescence of Drops on a Fluid Bath, Phys. Rev. Lett. 94, 177801 (2005).

[2] S. Protiere, A. Boudaoud, and Y. Couder, Particle-wave association on a fluid interface, J. Fluid Mech. 554, 85 (2006).

[3] A. Eddi, E. Sultan, J. Moukhtar, E. Fort, M. Rossi, and Y. Couder, Information stored in faraday waves: The origin of a path memory, J. Fluid Mech. 674, 433 (2011).

[4] J. Molacek and J. W. M. Bush, Drop bouncing on a vibrating bath, J. Fluid Mech. 727, 582 (2013).

[5] J. Moláček and J. W. M. Bush, Drops walking on a vibrating bath: Toward a hydrodynamic pilot-wave theory, J. Fluid Mech. 727, 612 (2013).

[6] P. A. Milewski, C. A. Galeano-Rios, A. Nachbin, and J. W. M. Bush, Faraday pilot-wave dynamics: Modeling and computation, J. Fluid Mech. 778, 361 (2015).
[7] L. Tadrist, J.-B. Shim, T. Gilet, and P. Schlagheck, Faraday instability and subthreshold faraday waves: Surface waves emitted by walkers, J. Fluid Mech. 848, 906 (2018).

[8] J. W. M. Bush, Pilot-wave hydrodynamics, Annu. Rev. Fluid Mech. 47, 269 (2015).

[9] E. Fort, A. Eddi, A. Boudaoud, J. Moukhtar, and Y. Couder, Path-memory induced quantization of classical orbits, Proc. Nat. Acad. Sci. USA 107, 17515 (2010).

[10] A. U. Oza, O. Wind-Willassen, D. M. Harris, R. R. Rosales, and J. W. M. Bush, Pilot-wave hydrodynamics in a rotating frame: Exotic orbits, Phys. Fluids 26, 082101 (2014).

[11] M. Labousse, A. U. Oza, S. Perrard, and J. W. M. Bush, Pilot-wave dynamics in a harmonic potential: Quantization and stability of circular orbits, Phys. Rev. E 93, 033122 (2016).

[12] S. Perrard, M. Labousse, M. Miskin, E. Fort, and Y. Couder, Self-organization into quantized eigenstates of a classical wavedriven particle, Nat. Commun. 5, 3219 (2014). 
[13] D. M. Harris, J. Moukhtar, E. Fort, Y. Couder, and J. W. M. Bush, Wavelike statistics from pilot-wave dynamics in a circular corral, Phys. Rev. E 88, 011001(R) (2013).

[14] P. J. Sáenz, T. Cristea-Platon, and J. W. M. Bush, Statistical projection effects in a hydrodynamic pilot-wave system, Nat. Phys. 14, 315 (2018).

[15] Y. Couder and E. Fort, Single-Particle Diffraction and Interference at a Macroscopic Scale, Phys. Rev. Lett. 97, 154101 (2006).

[16] A. Andersen, J. Madsen, C. Reichelt, S. R. Ahl, B. Lautrup, C. Ellegaard, M. T. Levinsen, and T. Bohr, Double-slit experiment with single wave-driven particles and its relation to quantum mechanics, Phys. Rev. E 92, 013006 (2015).

[17] G. Pucci, D. M. Harris, L. M. Faria, and J. W. M. Bush, Walking droplets interacting with single and double slits, J. Fluid Mech. 835, 1136 (2018).

[18] T. Bohr, Quantum physics dropwise, Nat. Phys. 14, 209 (2018).

[19] A. Eddi, E. Fort, F. Moisy, and Y. Couder, Unpredictable Tunneling of a Classical Wave-Particle Association, Phys. Rev. Lett. 102, 240401 (2009).

[20] A. Nachbin, P. A. Milewski, and J. W. M. Bush, Tunneling with a hydrodynamic pilot-wave model, Phys. Rev. Fluids 2, 034801 (2017).

[21] M. Hubert, M. Labousse, and S. Perrard, Self-propulsion and crossing statistics under random initial conditions, Phys. Rev. E 95, 062607 (2017).

[22] D. Shirokoff, Bouncing droplets on a billiard table, Chaos 23, 013115 (2013).

[23] T. Gilet, Dynamics and statistics of wave-particle interactions in a confined geometry, Phys. Rev. E 90, 052917 (2014).

[24] L. Tadrist, N. Sampara, P. Schlagheck, and T. Gilet, Interaction of two walkers: Perturbed vertical dynamics as a source of chaos, Chaos 28, 096113 (2018).

[25] T. Cristea-Platon, P. J. Sáenz, and J. W. M. Bush, Walking droplets in a circular corral: Quantisation and chaos, Chaos $\mathbf{2 8 ,}$ 096116 (2018).
[26] D. M. Harris and J. W. M. Bush, Generating uniaxial vibration with an electrodynamic shaker and external air bearing, J. Sound Vib. 334, 255 (2015).

[27] D. M. Harris, T. Liu, and J. W. M. Bush, A low-cost, precise piezoelectric droplet-on-demand generator, Exp. Fluids 56, 83 (2015).

[28] S. Protiere, Gouttes rebondissantes: une association ondeparticule à échelle macroscopique, Ph.D. thesis, Université Paris-Diderot-Paris VII, 2017.

[29] P. J. Sáenz, T. Cristea-Platon, and J. W. M. Bush, A hydrodynamic analog of Friedel oscillations, Sci. Adv. 6, eaay9234 (2020).

[30] G. Pucci, P. J. Sáenz, L. M. Faria, and J. W. M. Bush, Nonspecular reflection of walking droplets, J. Fluid Mech. 804, R3 (2016).

[31] C. Cohen-Tannoudji, B. Diu, F. Laloe, and B. Dui, Quantum Mechanics (Wiley-Interscience, Hoboken, NJ, 2006).

[32] M. Holland, S. Marksteiner, P. Marte, and P. Zoller, Measurement Induced Localization from Spontaneous Decay, Phys. Rev. Lett. 76, 3683 (1996).

[33] S. Kocsis, B. Braverman, S. Ravets, M. J. Stevens, R. P. Mirin, L. K. Shalm, and A. M. Steinberg, Observing the average trajectories of single photons in a two-slit interferometer, Science 332, 1170 (2011).

[34] B. Nowak, J. J. Kinnunen, M. J. Holland, and P. Schlagheck, Delocalization of ultracold atoms in a disordered potential due to light scattering, Phys. Rev. A 86, 043610 (2012).

[35] M. Arnal, G. Chatelain, M. Martinez, N. Dupont, O. Giraud, D. Ullmo, B. Georgeot, G. Lemarié, J. Billy, and D. Guéry-Odelin, Chaos-assisted tunneling resonances in a synthetic floquet superlattice, arXiv:2003.10387.

[36] A. Shudo and K. S. Ikeda, Complex Classical Trajectories and Chaotic Tunneling, Phys. Rev. Lett. 74, 682 (1995).

[37] S. Keshavamurthy and P. Schlagheck, Dynamical Tunneling: Theory and Experiment (CRC Press, Boca Raton, FL, 2011). 